\title{
ADRB2 polymorphism predicts FGID diagnoses and quality of life
}

Genetic variation in ADRB2 (encoding $\beta-2$ adrenergic receptor) is a predictor of diagnosis of a functional gastrointestinal disorder (FGID), and in some instances severity of bowel symptoms and poorer health-related quality of life (HRQOL).

$A D R B 2$ is widely expressed in the gastrointestinal tract and central nervous system, with a role in pain sensitivity. "We thus examined single nucleotide polymorphisms within the coding region of $A D R B 2$ as a potential factor that might account for some of these well-described relationships between FGIDs, extraintestinal pain symptoms, and mood features," explains author Gregory Sayuk.

Genotype analysis was performed in 170 individuals with FGIDs (such as IBS or functional dyspepsia) and 228 healthy individuals as controls. The researchers found that the rs 1042714 minor G allele was associated with a diagnosis of FGID, particularly functional dyspepsia. Moreover, G allele carriers had greater numbers of extraintestinal functional disorders and poorer HRQOL and, for those with IBS specifically, more severe bowel symptoms.

Sayuk proposes further work as individuals with the minor allele genotype "might have symptoms that are particularly driven by enhanced autonomic tone", speculating that they would be ideal candidates for pharmacotherapeutic approaches that temper these sympathetic responses (such as nonselective $\beta$-blockers).

Katrina Ray

Original article Kushnir, V. M. et al. Genetic variation in the beta-2 adrenergic receptor (ADRB2) predicts functional gastrointestinal diagnoses and poorer health-related quality of life. Aliment. Pharm. Ther. doi:10.1111/apt.12378 